\title{
Collapse of microchannels during anodic bonding: Theory and experiments
}

\author{
Wen-Pin Shih and Chung-Yuen Hui ${ }^{\text {a) }}$ \\ Department of Theoretical and Applied Mechanics, Cornell University, Ithaca, New York 14853 \\ Norman C. Tien \\ Berkeley Sensor and Actuator Center, Department of Electrical and Computer Engineering, \\ University of California, Davis, California 95616
}

(Received 13 October 2003; accepted 9 December 2003)

\begin{abstract}
By fabricating a large number of microchannels with different aspect ratios using anodic bonding, we demonstrate that a channel collapse can occur during anodic bonding as a result of the high electric field inside the channels. A theory is developed to establish a criterion for the collapse. This theory shows that the collapse can be avoided as long as the condition $\epsilon_{a} V^{2} a / E_{\text {eff }} d^{3}<1$ is satisfied, where $\epsilon_{a}$ is the permittivity of air, $V$ is the applied voltage for anodic bonding, $E_{\text {eff }}$ is a material constant which characterizes the elastic stiffness of the materials, $a$ is the half width of the channel, and $d$ is the channel depth. The validity of this theory is checked by comparing with experimental results. () 2004 American Institute of Physics. [DOI: 10.1063/1.1644898]
\end{abstract}

\section{INTRODUCTION}

Microfabrication techniques can provide potentially fast and efficient devices to separate DNA molecules. One example is the entropic trap array (ETA) by Han and Craighead. ${ }^{1}$ The basic design of the ETA is shown in Fig. 1. The array consists of alternating thin and thick regions in a microfabricated channel. The widths of these channels are denoted by $w_{t}$ and $w_{T}$, respectively. The depth of the thick channels $D$ is much greater than the radius of gyration of the DNA molecules. Inside the thick channels, the DNA molecules can achieve their equilibrium shapes which are approximately spherical. However, the depth of the thin channels, $d$, is smaller than the radius of gyration of the DNA molecules and can thus be regarded as molecular sieves. When driven by an electric field, DNA molecules are temporarily trapped at the entrance of these channels. As a result, the mobility of the DNA molecules in the thin channels is length dependent. It is this mobility dependence that allows the separation of DNA molecules. The ETA can also be used as an effective sample collection and launching device, as pointed out by Han and Craighead. ${ }^{1}$

The channels in the array are fabricated using photolithography and etching techniques on a silicon substrate of thickness $h_{s}$. Using anodic bonding, a Pyrex (Code 7740, Corning Inc., Corning, NY) glass of thickness $h_{g}$ is bonded to the silicon substrate [Fig. 1(a)]. One would expect that, for sufficiently small $d$, the electric force in the bonding process could pull the two surfaces of the thin channels into contact. Such contact poses a serious technical barrier to the fabrication of thinner channels. In the present work, detailed experiments are carried out to study the collapse of microchannels during bonding. Specifically, we determine the conditions of the channel collapse by subjecting channels with various depths and widths to different applied bias and temperature. A detailed electromechanical analysis is carried out to estab-

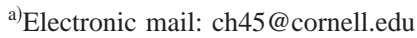

lish a criterion of contact. The theory is then compared with experimental observations.

The anodic bonding process was discovered by Pomerantz in $1968 .^{2}$ In this process, the two parts to be bonded (e.g., glass/silicon or glass/metal) is subjected to an applied dc voltage of about $1000 \mathrm{~V}$ and at $450^{\circ} \mathrm{C}$. It is commonly believed that bonding is facilitated by the high electric field on the glass/silicon interface and that this field is established by the migration of sodium ions to the cathode which reduces the potential drop across the glass layer. The sodium migration creates a polarized layer of negative ions adjacent to the anode/glass interface (Fig. 2). The electric field in a glass layer, sandwiched between two metal electrodes (Fig. 2 ), was analyzed by modeling the polarization region as a parallel plate capacitor, where most of the potential drop occurs. ${ }^{3-5}$ In their problem, there is no air gap since the glass and the metal substrate are assumed to be in intimate contact. This may not be the case in microchannels since much of the potential drop occurs inside the air gap and not in the polarization layer, as we shall demonstrate. An earlier theory, which takes into account the presence of the air gap, was performed by Anthony, who was interested in the effect of surface roughness on the bonding process. ${ }^{6} \mathrm{He}$ assumed that much of the potential drop occurs across the polarization layer since the height of the surface asperity is considerably less than the layer thickness. However, his final results concluded that most of the potential drop occurs across the air gap and the electric field in the air gap is given by $V / d$, where $V$ is the applied bias and $d$ is the thickness of the air gap. In this work, we revisit this problem without making explicit assumptions regarding the potential distribution between the air gap and the polarization layer. We also extend our analysis to semiconductor substrates, and this has not been analyzed before.

The electrostatic analysis must be coupled to a mechanical analysis of the contact condition. Although the bonding between rough surfaces and the collapse of microchannels have slightly different length scales, the underlying mechan- 

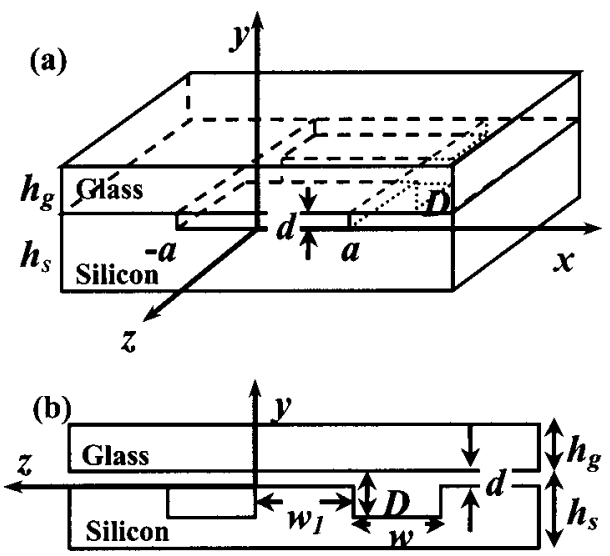

FIG. 1. Geometry of the microchannels in the entropic trap array [Fig. 1(a), three dimensional view; Fig. 1(b), view of a cross section in the $y z$ plane].

ics is similar. From this perspective, the present work is similar to a previous study carried out by Plaza et al. ${ }^{7}$ whose goal was to design a nondestructive testing technique to study the quality of anodic bonding. Rectangular cavities of various widths are fabricated on a silicon wafer. During bonding the cavity surface is subject to tensile stress generated by the electric field. If the electric field is sufficiently high, the stress brings the surfaces into contact. Bonding occurs due to increasing contact over time. The analogy between the problem considered by Plaza et al. ${ }^{7}$ and this present one is straightforward. The cavity in Plaza et al.'s experiments corresponds to the channel in our problem. The experiments of Plaza et al. indicate that bonding depends on the stiffness of the cavity. However, no theoretical analysis was carried out to quantify their depictions. Our goal is to obtain an expression relating the maximum deflection of the surfaces in terms of the geometry of the cavity, the deformation behavior of the glass and the silicon, as well as the applied voltage. The deformation of the glass was analyzed by Anthony. ${ }^{6} \mathrm{He}$ regarded the glass above the air gap as a slender beam. This is generally not a good approximation unless the thickness of the glass layer (millimeter) is much less than the width of the air gap (tens of nanometers) or the microchannels (tens of micrometers). In the present work, a different approach is used to estimate the deformation of both the silicon substrate and the glass wafer, and the analysis is experimentally verified.

\begin{tabular}{|c|c|}
\hline Metal cathode & 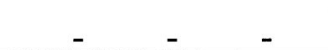 \\
\hline Nonpolarized gla & $\begin{array}{l}\mathrm{sa}^{+} \uparrow_{\mathrm{Na}^{+}} \mathrm{Na}^{+} \mathrm{Na}^{+} \uparrow_{\mathrm{Na}^{+}} \uparrow_{\mathrm{Na}^{+}}^{\mathrm{Na}^{+}} \\
\end{array}$ \\
\hline Polarized region & $\begin{array}{ccc}\mathrm{O}^{2-} & \mathrm{O}^{2-} & \mathrm{O}^{2-} \\
\mathrm{O}^{2-} & \end{array}$ \\
\hline Metal anode & $+\quad+$ \\
\hline
\end{tabular}

FIG. 2. Schematics of the migration of sodium ions inside the glass during anodic bonding. The glass is sandwiched by two metal electrodes where a bias is applied. A polarization region with negative oxygen ions forms as sodium ions migrate to the cathode.
The plan of this work is as follows. In Sec. II a model of the collapse is described. Since no amount of contact can be tolerated in practice, our goal is to establish a condition for the initial contact. In Sec. III, the potential drop across the air gap in a thin channel is estimated and expressed in terms of the applied voltage. The dependence of the potential drop on the temperature is also studied. Comparisons are made with previous results in anodic bonding. In Sec. IV, the electric field acting on the walls of the thin channels is related to the potential drop, and the deformation of the thin channels is computed. In particular, an estimate of the maximum deflection or displacement of the channel walls is given in closed form. Closed-form analytical expressions for the contact condition are also given. The concept of the contact map is introduced. Details of the experiments and the comparison with theory are given in Sec. V. The article ends with a discussion of anodic bonding and a summary of results.

\section{MODEL FOR CONTACT}

The geometry is shown in Figs. 1(a) and 1(b). The thickness of the silicon wafer $h_{s}(\approx 0.5 \mathrm{~mm})$ and the Pyrex glass $h_{g}(\approx 0.5 \mathrm{~mm})$ are assumed to be much greater than the channel depths, $D(\approx 100 \mathrm{~nm})$ and $d(\approx 30 \mathrm{~nm})$. The silicon is assumed to be linearly elastic with Young's modulus $E_{s}$ and Poisson's ratio $\nu_{s}$. The glass is assumed to be linearly viscoelastic with a time independent Poisson's ratio $\nu_{g}$. The creep function in tension of the glass is denoted by $C(t)$. The strain tensor $\epsilon_{i j}$ is related to the stress tensor $\sigma_{i j}$ through the Boltzmann superposition principle, ${ }^{8}$ i.e.,

$$
\epsilon_{i j}=\left(1+\nu_{g}\right) C * \sigma_{i j}+\nu_{g} C * \sigma_{k k} \delta_{i j} .
$$

The operator $C * \sigma_{i j}$ is defined by

$$
C * \sigma_{i j} \equiv \int_{0^{-}}^{t} C(t-\tau) \partial \sigma_{i j} / \partial \tau d \tau,
$$

where $0^{-}$allows for the possibility of a sudden initial step load. In the following, an explicit solution is specifically given for a Maxwell material although the analysis is valid for arbitrary creep functions. ${ }^{8}$ The creep function for a Maxwell material is

$$
C(t)=\left(1 / E_{g}\right)+(t / \eta)
$$

where $E_{g}$ is the short time tensile modulus and $\eta$ is the viscosity. The special case of a linearly elastic material corresponds to $\eta \rightarrow \infty$.

\section{DETERMINATION OF ELECTRIC FIELD}

Since the width $2 a$ of a typically thin channel is much greater than its depth, the electric field is uniform except at the edges. Therefore, the electric field is assumed to be independent of the $x$ and $z$ coordinates and the fields are estimated by solving the problem in Fig. 3. The silicon wafer is grounded at the bottom surface and a negative bias $V<0$ is applied to the glass. Following Albaugh's work, ${ }^{5}$ the glass layer is divided into two layers. The first layer, of thickness $y_{p}$, is the polarization region with a volume concentration of anions, denoted by $\sigma_{p}<0$. The flow of sodium ions to the cathode reduces the potential drop across the nonpolarized 


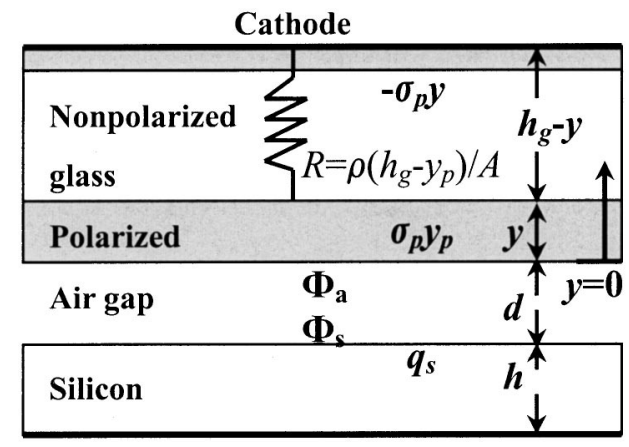

Anode

FIG. 3. Schematics of the model to compute the potential drop across the air gap. The thickness of the polarization layer is $y_{p}$. Sodium ion flow outside the polarization layer obeys Ohm's law. Poisson's equation is solved to obtain potential drop inside the polarization layer assuming the charge density of oxygen ions is uniform.

region, leading to the growth of the polarized layer. Two assumptions have been made for the polarization of glass. 9,10 First, the electrodes do not provide any ions to the glass. Second, only positive ions are mobile. Ion flow ceases when the full potential drop occurs across the polarized region and the air gap. The polarized layer is modeled as a linear dielectric material with permittivity $\epsilon_{g}$. The nonpolarized glass layer is treated as a linear ohmic material and is represented schematically as a resistor (Fig. 3). The effect of cathode blocking due to the accumulation of sodium ions close to the cathode is modeled by an infinitesimally thin layer of sodium ions with areal charge density $-\sigma_{p} y_{p}$ (Fig. 3).

Denote the surface potential of the glass/air interface and air/silicon interface by $\Phi_{a}$ and $\Phi_{s}$, respectively, the potential drop across the thin channel $\Delta \Phi$ is

$$
\Delta \Phi=\Phi_{s}-\Phi_{a}=q_{s} d / \epsilon_{a},
$$

where $\epsilon_{a}$ is the permittivity of air and $q_{s}$ is the surface charge per unit area of the silicon wafer. To compute the potential drop across the polarization region, we solve the Poisson's equation

$$
d^{2} \Phi / d y^{2}=-\sigma_{p} / \epsilon_{g},
$$

where $\Phi(y)$ is the potential and $\sigma_{p}$ is the charge concentration inside the region. Continuity of the electric displacement across the lower glass surface at $y=0$ gives the boundary condition

$$
d \Phi /\left.d y\right|_{y=0}=-q_{s} / \epsilon_{g} .
$$

Also, we enforce the condition that

$$
\Phi(y=0)=\Phi_{a} .
$$

The solution of Eq. (5a) subjected to the boundary conditions $(5 b)$ and $(5 c)$ is then

$$
\Phi=\Phi_{s}-\Delta \Phi-\left(y / \epsilon_{g}\right)\left[q_{s}+\left(\sigma_{p} y / 2\right)\right],
$$

where $\Phi_{a}=\Phi_{s}-\Delta \Phi$. Using Eqs. (4) and (6), the potential drop across the nonpolarized region, $\Phi\left(y_{p}\right)-V$, is

$$
\Phi\left(y_{p}\right)-V=\Phi_{s}-\Delta \Phi-\left(y_{p} / \epsilon_{g}\right)\left[q_{s}+\left(\sigma_{p} y_{p} / 2\right)\right]-V .
$$

Using Gauss's law, the potential drop across the nonpolarized region is also given by

$$
\Phi\left(y_{p}\right)-V=\left(h_{g}-y_{p}\right)\left(q_{s}+\sigma_{p} y_{p}\right) / \epsilon_{g} .
$$

Since $q_{s}$ is related to $\Delta \Phi$ by Eq. (4), we solve the potential drop across the air gap by equating the right-hand sides of Eqs. (7a) and (7b). This is found to be

$$
\Delta \Phi=-(1+\beta)^{-1} V\left(1-\phi_{s}+\alpha \delta-\alpha \delta^{2} / 2\right),
$$

where $\phi_{s}=\Phi_{s} / V$ is the normalized surface potential of the silicon and $\delta=y_{p} / h_{g}$ is the normalized thickness of the polarized layer. The dimensionless parameters $\alpha$ and $\beta$ are defined by $\alpha=h_{g}^{2} \sigma_{p} / \epsilon_{g} V$ and $\beta=\epsilon_{a} h_{g} / \epsilon_{g} d$. Note that if there were no ion flow, i.e., $\alpha=0$, then $\Delta \Phi=-V\left(1-\phi_{s}\right) /(1$ $+\beta)$. This solution corresponds to the case where the glass is an ideal dielectric. Since $\beta$ is typically much greater than 1 , the potential drop across the air gap is very small without ion flow.

To determine $\delta$, we compute the current density $J$ in the glass using Ohm's law

$$
J=-\sigma_{p} d y_{p} / d t=\left[\Phi\left(y_{p}\right)-V\right]\left(h_{g}-y_{p}\right)^{-1} / \rho,
$$

where $\rho$ is the resistivity of glass. Using Eqs. (4), (6) and (8), (9) can be rewritten as

$$
d \delta / d \tau=-\beta(1+\beta)^{-1}\left[-\alpha^{-1}\left(1-\phi_{s}\right)+\delta^{2} / 2+\beta^{-1} \delta\right],
$$

where $\tau=t /\left(\epsilon_{g} \rho\right)$ is a normalized time. The initial condition of Eq. (10) is $\delta(\tau=0)=0$ since the initial thickness of the polarized layer is zero. Equation (10) can be solved in closed form by assuming that the surface potential of the silicon is much smaller than the bonding bias, i.e., $\phi_{s}=\Phi_{s} / \ll 1$, and thus treating the silicon as a conductor. This assumption will be fully justified in the Appendix. The normalized thickness of the polarized layer is found to be

$$
\delta=\beta^{-1}\left\{\omega\left[1-C e^{-\omega \tau /(1+\beta)}\right]\left[1+C e^{-\omega \tau /(1+\beta)}\right]^{-1}-1\right\},
$$

where $\omega \equiv \sqrt{1+2 \beta^{2} / \alpha}$ and $C \equiv(\omega-1) /(\omega+1)$. Using $\epsilon_{a}$ $=8.85 \times 10^{-12} \mathrm{~F} / \mathrm{m}, \quad \epsilon_{g}=10 \epsilon_{a},{ }^{4} \quad V=-10^{3} \mathrm{~V}, \quad \sigma_{p}$ $=-2.72 \times 10^{8} \mathrm{C} / \mathrm{m}^{3}, h_{g}=0.5 \mathrm{~mm}$, and $d=100 \mathrm{~nm}$, we found $\alpha \cong 7.70 \times 10^{8}, \beta \cong 500.00, \omega \cong 1+3.25 \times 10^{-4}$, and $C \cong 1.62 \times 10^{-4}$. This example shows that an excellent approximation for the potential drop across the air gap can be obtained by assuming that $\alpha \gg 1$ and $\beta \gg 1$ in Eq. (11). That is, $\delta$ is typically much less than unity so that the $\delta^{2}$ term in Eq. (8) is negligible in comparison with $\delta$. Therefore, Eq. (8) is well approximated by

$$
\Delta \Phi=-V(1+\alpha \delta)(1+\beta)^{-1} .
$$

Two special cases are of practical interest. For microchannels, the condition $\beta^{2} / \alpha \ll 1$ is usually satisfied (see the earlier example), so both $\omega$ and $C$ are much less than unity. In this case, $\omega \rightarrow 1+\beta^{2} / \alpha$ and $C \rightarrow \beta^{2} / 2 \alpha$, and Eqs. (11) and (12) simplify to

$$
\delta \cong \beta \alpha^{-1}\left[1-e^{-\omega \tau /(1+\beta)}\right] \approx \beta \alpha^{-1}\left(1-e^{-\tau / \beta}\right)
$$




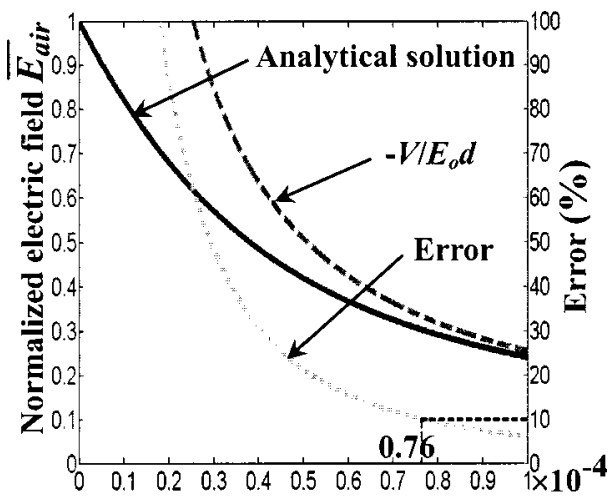

Normalized air gap thickness $(1 / \beta)$

FIG. 4. Normalized steady state electric field across the air gap $\bar{E}_{\text {air }}$ $=\Delta \Phi(\tau \rightarrow \infty) / E_{0} d$ vs normalized air gap thickness $1 / \beta=\epsilon_{g} d / \epsilon_{a} h_{g}$. $E_{0}=-V \epsilon_{g}(1+\sqrt{2 \alpha}) / \epsilon_{a} h_{g}$ is the maximum electric field across the air gap which occurs at zero channel thickness $(d=0)$. The dashed line is $\bar{E}_{\text {air }}=-V / E_{0} d$. At $1 / \beta=0.76 \times 10^{-4}$ (or $d>3.8 \mathrm{~nm}$ ), the error between the dashed line and the solid line is $10 \%$.

$$
\begin{aligned}
\Delta \Phi & =-V(1+\beta)^{-1}\left\{1+\beta\left[1-e^{-\omega \tau /(1+\beta)}\right]\right\} \\
& \approx-V\left[1-e^{-\tau / \beta}\right]
\end{aligned}
$$

since $\beta \gg 1$. Equation (13b) shows that, for $\tau>\beta$ or $t$ $>\rho \epsilon_{a} h_{g} / d \approx 0.4 \mathrm{~ms}$, where we use $\rho \cong 10^{6} \Omega \mathrm{cm}, h_{g}$ $=500 \mu \mathrm{m}$, and $d=100 \mathrm{~nm}$, most of the applied bias drops across the air gap instead of the polarization region. The second case corresponds to the anodic bonding where $d$ $\cong 0$. In this case, $\beta^{2} / \alpha \rightarrow \infty$ so that $\omega / \beta \rightarrow \sqrt{2 / \alpha} \ll 1$ and $C$ $\rightarrow 1$, and Eq. (11) reduces to

$$
\delta=\sqrt{2 / \alpha} \tanh (\tau / \sqrt{\alpha / 2}) .
$$

Equation (14) differs from Albaugh's expression ${ }^{5}$ for $\delta$ by a factor of $\sqrt{2}$. This discrepancy is due to the fact that the polarization region is approximated by a parallel plate capacitor by Albaugh. ${ }^{5}$ Equation (14) provides a slightly more accurate description of the polarization thickness during anodic bonding. The polarization region for the case of $d=0$ is fully developed when $\tau \geqslant \sqrt{\alpha / 2}$, which is about $1 \mathrm{~s}$ in real time.

The steady state electric field across the air gap is $E_{\text {air }}$ $=\Delta \Phi(\tau \rightarrow \infty) / d$. The normalized steady state electric field

$$
\begin{aligned}
\bar{E}_{\mathrm{air}} & =E_{\mathrm{air}} / E_{0} \\
& =\left(1+\alpha \beta^{-1} \sqrt{1+2 \beta^{2} \alpha^{-1}}-\alpha \beta^{-1}\right) /(1+\sqrt{2 \alpha})
\end{aligned}
$$

is plotted against the normalized air gap thickness $1 / \beta$ in Fig. 4. $E_{0}$ is the maximum electric field across the air gap. This maximum occurs at zero channel thickness $(d=0)$ and is found to be $-V(1+\sqrt{2 \alpha}) / d(1+\beta)$ using Eqs. (12) and (14). The parameters used in Fig. 4 are $V=-10^{3} \mathrm{~V}, \epsilon_{g}$ $=10 \epsilon_{a}, h_{g}=0.5 \mathrm{~mm}$, and $\alpha=7.70 \times 10^{8}$. Figure 4 shows that steady state electric field is well approximated by $-V / d$ as long as $1 / \beta>0.76 \times 10^{-4}$ (or $d>3.8 \mathrm{~nm}$ ). Thus, for all practical purpose, the steady state electric field across a microchannel is $-V / d$.

For microchannels, Eq. (13b) shows that the normalized characteristic time required to reach full surface potential is

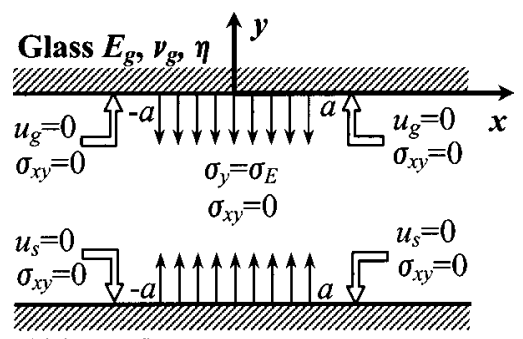

Silicon $E_{s}, \nu_{s}$

FIG. 5. Traction and the displacement boundary conditions imposed on the surface of the glass and silicon. The shear stress $\sigma_{x y}$ and the vertical displacement are zero along the bonding interfaces.

$\beta$ whereas for anodic bonding (with no air gap) this time is $\sqrt{\alpha / 2}$ [see Eq. (14)]. Using the same parameters as before, $\beta / \sqrt{\alpha / 2} \approx 0.0255$. Therefore, the time needed to establish a complete polarization region is two orders of magnitude less in the presence of an air gap. This is because the potential drop across the latter is closed to the applied bias; resulting in a much thinner polarization layer (about 65 times thinner) which needs much less time to develop. Using $d=100 \mathrm{~nm}$ and $\rho=10^{6} \Omega \mathrm{cm}$, the polarization region is fully developed in about $0.4 \mathrm{~ms}$ which is negligible compared to typical bonding times (minutes). Thus, for all practical purposes, the potential drop across the air gap can be taken to be the applied bias.

\section{DEFORMATION OF THE THIN CHANNEL}

Since the semiconductor can be treated as a perfect conductor, the electric stress $\sigma_{E}$ is the total normal force per unit area acting on the channel walls given by

$$
\sigma_{E}=\epsilon_{a}(\Delta \Phi)^{2} /\left(2 d^{2}\right) \text {. }
$$

For $d>1 \mathrm{~nm}\left(\beta^{2} / \alpha \ll 1\right), \Delta \Phi$ is given by Eq. (13b), i.e.,

$$
\sigma_{E}=\left(\epsilon_{a} V^{2} / 2 d^{2}\right)\left(1-\exp \left[-d t / \rho \epsilon_{a} h_{g}\right]\right)^{2} .
$$

By symmetry, the maximum displacement $\nu_{\max }$ occurs at the center of the thin channel [Fig. 1(a)]. Since the width $2 a$ of the thin channel is much greater than the air gap $d$ [Fig. $1(\mathrm{a})]$, the deformation of the walls of the thin channels can be estimated by computing the displacement of a channel of infinite length in the $z$ direction. The deformation is thus in a state of plane strain. In addition, since the thickness of the silicon and the glass is much greater than $d$, the glass and the silicon can be assumed semi-infinite. The displacements of the upper (glass) and lower (silicon) wall can be obtained by solving the boundary value problems in Fig. 5, where it is assumed that the shear traction and the vertical displacement vanish along the silicon/glass interface, i.e., on $y=0$ and $|x|>a$. The vertical displacement of the silicon wall $\nu_{s}$ of the channel (positive downwards) is found to be $\mathrm{e}^{11}$

$$
\nu_{s}=2\left(1-\nu_{s}^{2}\right) \sigma_{E} \sqrt{a^{2}-x^{2}} / E_{s}, \quad|x|<a .
$$

Since the glass layer is viscoelastic, the vertical displacement of the glass wall $\nu_{g}$ of the channel (positive downwards) is dependent on the loading history. Using the correspondence principle of linear viscoelasticity, ${ }^{8}$ it is found to be 


$$
\nu_{g}=2\left(1-\nu_{g}^{2}\right) \sqrt{a^{2}-x^{2}} \int_{0^{-}}^{t} C(t-\tau) \sigma_{E}(\tau) d \tau .
$$

Since the relaxation time of the glass is much longer than $\beta \rho \epsilon_{g}$ which is on the order of a millisecond so that

$$
\sigma_{E}=\epsilon_{a} V^{2} / 2 d^{2} .
$$

Furthermore, the biased voltage is typically maintained at a constant level $V$ for all $t<t_{\text {end }}$, where $t_{\text {end }}$ is the time of the application of voltage. For $t>t_{\text {end }}$, the applied voltage is zero. In this case, Eq. (16b) is

$$
\begin{aligned}
& \nu_{g}=2\left(1-\nu_{g}^{2}\right) \sqrt{a^{2}-x^{2}} \sigma_{E} C(t), \quad t \leqslant t_{\text {end }}, \\
& \nu_{g}=2\left(1-\nu_{g}^{2}\right) \sqrt{a^{2}-x^{2}} \sigma_{E}\left[C(t)-C\left(t-t_{\text {end }}\right)\right], \\
& t>t_{\text {end }} .
\end{aligned}
$$

According to Eqs. (17a) and (17b), the maximum displacement occurs at $t_{\text {end }}$ so that it is only necessary to consider $t$ $\leqslant t_{\text {end }}$. In particular, the maximum displacement is directly proportional to the creep compliance. For a Maxwell material, Eqs. (17a) and (17b) are

$$
\begin{gathered}
\nu_{g}=2\left(1-\nu_{g}^{2}\right)\left(\sigma_{E} / E_{g}\right)\left[1+\left(E_{g} t / \eta\right)\right] \sqrt{a^{2}-x^{2}}, \\
t \leqslant t_{\text {end }}, \\
\nu_{g}=2\left(1-\nu_{g}^{2}\right) \eta^{-1} \sigma_{E} t_{\text {end }} \sqrt{a^{2}-x^{2}}, \quad t>t_{\text {end }},
\end{gathered}
$$

respectively. Thus, for a Maxwell solid, the displacement is directly proportional to time.

The maximum total displacement of the channel wall is

$$
\nu_{\max }=\nu_{s}(x=0)+\nu_{g}(x=0, t) .
$$

Using Eqs. (16a) and (17a), this is

$$
\nu_{\max }=2 \sigma_{E} a E_{\mathrm{eff}}^{-1}+2 \sigma_{E} a t \eta^{-1},
$$

where $E_{\text {eff }}^{-1}=\left(1-\nu_{s}^{2}\right) / E_{s}+\left(1-\nu_{g}^{2}\right) / E_{g}$. Contact occurs when $\nu_{\max }=d$, i.e.,

$$
t_{\text {contact }}=\left(\eta d / 2 \sigma_{E} a\right)\left[1-2 \sigma_{E} a\left(E_{\text {eff }} d\right)^{-1}\right] .
$$

From Eq. (20a), it is seen that contact occurs instantaneously if

$$
2 \sigma_{E} a / E_{\text {eff }} d \geqslant 1 .
$$

Therefore, Eq. (22) is the elastic contact condition for a material with infinite viscosity. To avoid contact, it is necessary and sufficient that

$$
t_{\text {contact }}=\left(\eta d / 2 \sigma_{E} a\right)\left[1-2 \sigma_{E} a\left(E_{\text {eff }} d\right)^{-1}\right]>t_{\text {end }} .
$$

Substituting $\sigma_{E}$ of Eq. (16c) into Eq. (23), the condition for noncontact is then

$$
\eta d^{3}\left(a \epsilon_{a} V^{2} t_{\text {end }}\right)^{-1}\left[1-\epsilon_{a} V^{2} a\left(E_{\text {eff }} d^{3}\right)^{-1}\right]>1 .
$$

Note that the elastic contact condition (22), i.e.,

$$
\epsilon_{a} V^{2} a \geqslant E_{\text {eff }} d^{3},
$$

is included in Eq. (24) since elastic contact implies that the left-hand side of Eq. (24) is negative. Because the contact condition depends on $d^{3}$, a slight decreases in the air gap can cause contact. Note also that the contact condition is inde-

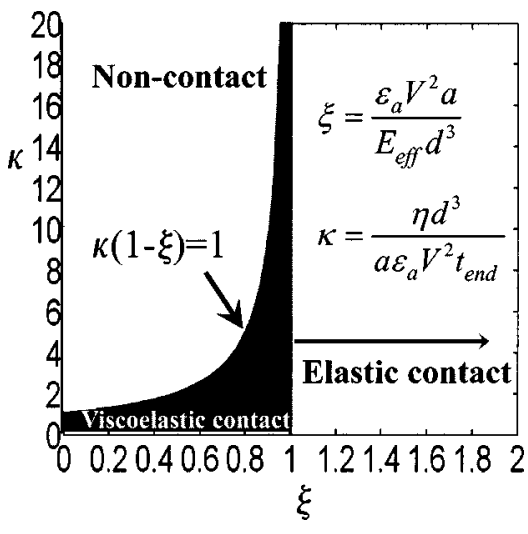

FIG. 6. Contact map. There is no contact if $(\xi, \kappa)$ lies above the curve $\kappa(1-\xi)=1$. Elastic contact occurs if $\xi \geqslant 1$. Viscoelastic contact occurs in the region $\kappa(1-\xi)=1$ and $\xi<1$.

pendent of the thickness of the glass and silicon layer, as long as these are large compared with the air gap.

We are now in a position to address the following question: how should viscosity (or temperature), electric permittivity, geometry, bonding time, bonding voltage, and elastic modulus be chosen so that contact can be avoided? The solution can be summarized by a two parameter contact map. The basic idea is to define a dimensionless function

$$
\Omega(\kappa, \xi)=\kappa(1-\xi),
$$

where $\kappa$ and $\xi$ are dimensionless material parameters defined by

$$
\begin{aligned}
& \kappa=\eta d^{3} / a \epsilon_{a} V^{2} t_{\mathrm{end}}, \\
& \xi=\epsilon_{a} V^{2} a / E_{\mathrm{eff}} d^{3},
\end{aligned}
$$

respectively. According to Eq. (26a), noncontact is possible if $(\kappa, \xi)$ lies in the region $\Omega(\kappa, \xi)>1$. This is the shaded region shown in Fig. 6. Figure 6 shows that there are three distinct regions in the parameter plane $(\kappa \geqslant 0, \xi \geqslant 0)$. There is no contact when $(\kappa, \xi)$ lies in the region above the curve $\kappa=1 /(1$ $-\xi), 0 \leqslant \xi<1$. Viscoelastic contact occurs when $(\kappa, \xi)$ lies below this region. Elastic contact occurs only when $\xi \geqslant 1$.

As an example, lowering the voltage increases $\kappa$, thus reducing the possibility of contact. However, lowering the voltage also increases $t_{\text {end }}$, the time to complete anodic bonding. According to Eq. (26b), the relevant physical parameter is $V^{2} t_{\text {end }}$. Specifically, lowering this parameter reduces the likelihood of contact. Another way of reducing the chance of contact is to increase the viscosity by lowering the bonding temperature. For Pyrex glass, the following empirical relation is known:

$$
\eta=10^{C_{1}+\left[C_{2} / T\left({ }^{\circ} \mathrm{C}\right)+C_{3}\right]} \text { (Pa s), }
$$

where $C_{1}, C_{2}$, and $C_{3}$ are material constants. ${ }^{12}$ These constants can be determined by fitting measured viscosity versus temperature data ${ }^{13}$ and it is found that $C_{1}=-8.73, C_{2}$ $=15365.7$, and $C_{3}=181.1$. At $450^{\circ} \mathrm{C}$, the viscosity of glass is found to be $4.1 \times 10^{15} \mathrm{~Pa} \mathrm{~s}$. At this temperature, the deformation due to viscous flow is very small compared with elastic deformation unless the bonding time exceeds $\eta / E_{g}$ $\approx 10^{5} \mathrm{~s} \cong 28 \mathrm{~h}\left(E_{g}=62.7 \mathrm{GPa}\right)$. 


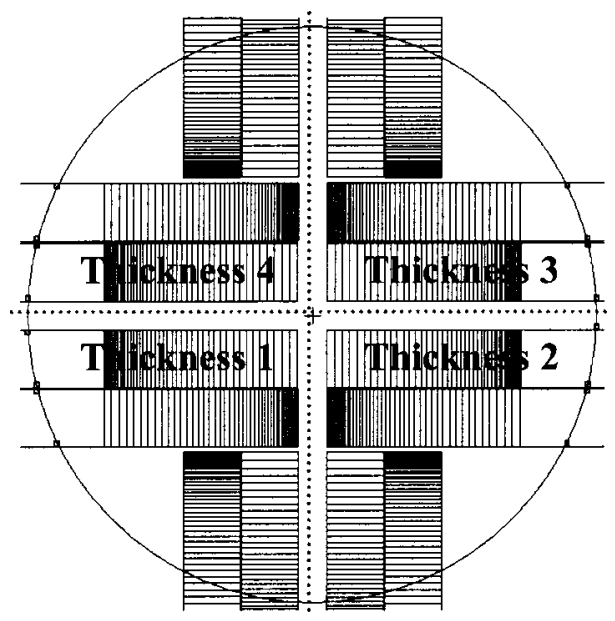

FIG. 7. Wafer-level layout of microchannels. The circle denotes the edge of the silicon wafer, which is separate into four regions by the dotted lines. Each solid line represents a single microchannel. Channels in each region have the same depth but different widths with $4 \mu \mathrm{m}$ increments.

\section{EXPERIMENTS}

To determine the applicability of the collapse condition (25), a large number of channels with different width-todepth ratio $2 a / d$ are patterned on a silicon wafer using photolithography. Wafer thickness is approximately $500 \mu \mathrm{m}$. Figure 7 shows the wafer-level layout of the microchannels. The silicon wafer is divided into four regions. Within each region, the channel depth is identical. The depth of the microchannels is measured using a Dektak 3030 profilometer. There are 50 different channel widths within each region. The range of the widths is between 3 and $199 \mu \mathrm{m}$ with $4 \mu \mathrm{m}$ increments. This allows us to pin point the critical collapse channel width. A summary of the microchannel dimensions are given in Table I. To approximate the plane strain condition, all microchannels are $1 \mathrm{~cm}$ long. The spacing between each of the two adjacent channels is six times the narrower of the two (Fig. 8). This spacing mechanically isolates the channels from each other so that Eq. (25), which is based on an isolated channel, applies. We have performed a finite element analysis to verify that this separation is indeed sufficient to ensure mechanical isolation. To eliminate the possibility of internal air pressure, which resists the collapse of the channels, the ends of all the microchannels are connected by a pair of $5 \mu \mathrm{m}$ wide channels to the edge of the wafer, thus providing an escape path for trapped air. The micro-
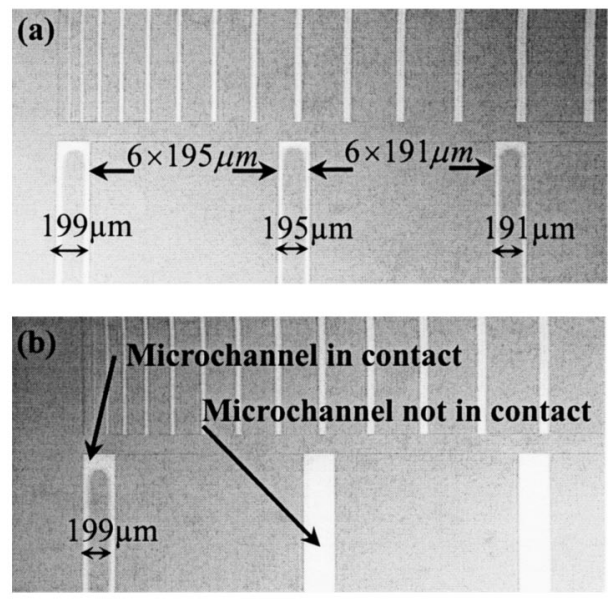

FIG. 8. Images of microchannels in contact. Channels turn from bright to gray when they are in contact. Bonding parameters are $1027 \mathrm{~V}, 450{ }^{\circ} \mathrm{C}$, and $30 \mathrm{~min}$. The depth of the microchannels is $1683 \AA$ in Fig. 8(a) and $2066 \AA$ in Fig. 8(b).

channels in the four regions are dry-etched using $\mathrm{SF}_{6}: \mathrm{O}_{2}$ $(15: 1 \mathrm{sccm})$ at 0.150 Torr and $200 \mathrm{~W}$ for four different durations. The etch rate is about $550 \AA / \mathrm{min}$.

The patterned wafer is then anodically bond under atmospheric conditions to a Pyrex glass of $0.5 \mathrm{~mm}$ thickness. The bonding parameters are given in Table I. Before bonding, both Pyrex and silicon wafers are cleaned using Pirahna $\left(\mathrm{H}_{2} \mathrm{SO}_{4}: \mathrm{H}_{2} \mathrm{O}_{2}=3: 1\right)$ at $100^{\circ} \mathrm{C}$ for $30 \mathrm{~min}$ to remove surface organics and metals.

The bonded wafers are inspected under a microscope. Figure 8(a) shows the image of a $199 \mu \mathrm{m}$ wide channel loaded at the critical load for channel collapse. The channels, shown as isolated rectangles are bright before contact. Figure 8 shows the onset of the channel collapse with increasing width and decreasing depth. In a given channel, once the roof makes contact, it collapses quickly under the influence of attractive interfacial surface forces, ceasing only when a narrow "moat" of the noncontacted region is left. The microchannel turns from bright to gray as it collapses.

Two bonding duration (60 and $30 \mathrm{~min}$ ) are imposed on identical specimens under identical bonding conditions [wafer 1 and wafer 2 in Table I]. There is no observable difference between the results. This result can be explained by comparing the $t_{\text {end }} / \eta$ and the $1 / E_{\text {eff }}$ terms in Eq. (20a). At $450{ }^{\circ} \mathrm{C}$, the viscosity of the Pyrex is $4.1 \times 10^{15} \mathrm{~Pa}$ s so that the $t_{\text {end }} / \eta$ term is at least two orders of magnitude smaller than

TABLE I. Designed bonding parameters and channel depths.

\begin{tabular}{ccccc}
\hline \hline Wafer & $\begin{array}{c}\text { Bonding } \\
\text { voltage } \\
(\mathrm{V})\end{array}$ & $\begin{array}{c}\text { Bonding } \\
\text { temperature } \\
\left({ }^{\circ} \mathrm{C}\right)\end{array}$ & $\begin{array}{c}\text { Bonding } \\
\text { time } \\
(\mathrm{min})\end{array}$ & $\begin{array}{c}\text { Measured channel depth } \\
(\AA)\end{array}$ \\
\hline 1 & 1327 & 450 & 60 & $1863 \pm 1,2066 \pm 155,2420 \pm 73,2610 \pm 24$ \\
2 & 1327 & 450 & 30 & $1863 \pm 1,2066 \pm 155,2420 \pm 73,2610 \pm 24$ \\
3 & 1022 & 450 & 30 & $552 \pm 6,749 \pm 13,935 \pm 21,1105 \pm 38$ \\
4 & 1022 & 400 & 30 & $484 \pm 20,758 \pm 19,1053 \pm 28,1316 \pm 34$ \\
5 & 1022 & 350 & 30 & $450 \pm 41,723 \pm 39,930 \pm 18,1144 \pm 76$ \\
\hline \hline
\end{tabular}




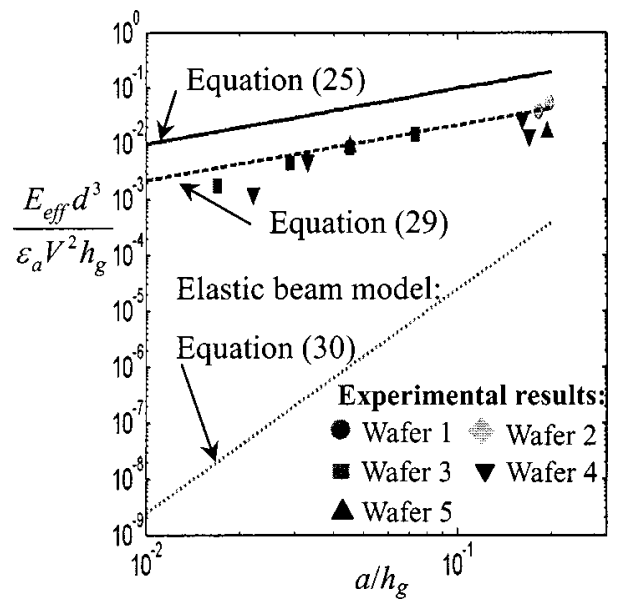

FIG. 9. Comparison of experimental data with theory. Equation (28) is plotted using $a_{c}$ obtained from experiments. Different symbols correspond to data from different wafers. The analytical result [Eq. (25)] is shown as a solid line. The modified analytical result [Eq. (29)] is shown as a dashed line. The analytical results of Anthony (see Ref. 6) based on beam theory is shown as a dotted line.

the $1 / E_{\text {eff }}$ term. Therefore, the effect of viscous flow is negligible at $450{ }^{\circ} \mathrm{C}$. Fortunately, bonding at higher temperatures is not preferred in practice since the metal surface of the hot plate of the bonding systems can be severely oxidized.

According to Eq. (25), the onset of the channel collapse satisfies

$$
E_{\text {eff }} d^{3} /\left(\epsilon_{a} V^{2} h_{g}\right)=a_{c} / h_{g},
$$

where $a_{c}$ is the critical channel width at the onset of the collapse. Both sides of the earlier equation can be evaluated using the experimentally determined critical channel width $a_{c}$ and the corresponding channel depth. The experimental determined $E_{\text {eff }} d^{3} / \epsilon_{a} V^{2} h_{g}$ vs $a_{c} / h_{g}$ is shown in Fig. 9 and the present theory [Eq. (25)] is also plotted as a comparison. The effective modulus, $E_{\text {eff }}$ is obtained using Eq. (20b) with $E_{s}$ $=180 \mathrm{GPa}, \nu_{s}=0.22, E_{g}=67.2 \mathrm{GPa}$, and $\nu_{g}=0.22$ and is found to be $51 \mathrm{GPa}$. The experimental data shows that Eq. (25) provides the correct scaling law but overestimates $E_{\text {eff }} d^{3} / \epsilon_{a} V^{2} h_{g}$ for a given critical channel width. We believe the cause of this discrepancy is due to an overestimate of the electric force acting on the channel. Indeed, the electric stress on the channel walls is computed based on the maximum potential drop $\Delta \Phi=-V$, whereas, in practice, a significant potential drop can occur across the glass wafer or across the silicon surface. For example, in our experiment, sodium electroplating was observed on the cathode (Fig. 10). Our model assumes that all the sodium ions stay on the glass side of the glass/cathode interface so that the full potential can occur across the polarization region and the air gap. However, it has been shown that a significant potential drop can occur due to the overpotential for the crystal growth of the sodium. ${ }^{4,5}$ Second, the native oxide (typically about 16 $\mathrm{nm}$ ) and oxidation of the silicon surface at high temperature and high electric fields can also compensate part of the applied bias. For example, the formation of a 50-nm-thick oxide layer in the anode was observed by Wallis after anodic bonding. ${ }^{14}$ There are also other minor effects which can fur-

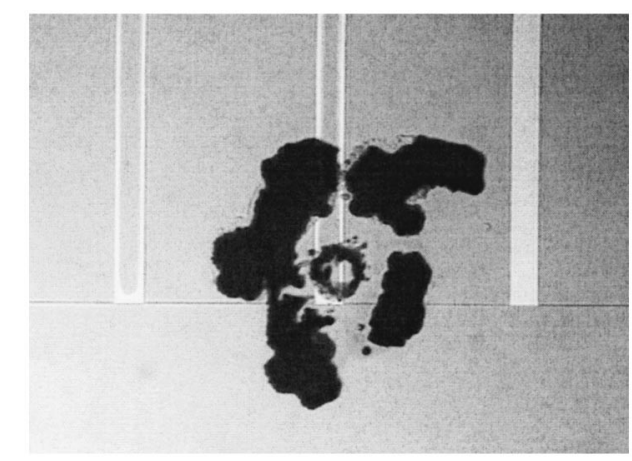

FIG. 10. Image of electroplated sodium crystals at the cathode after anodic bonding.

ther reduced the potential drop across the air gap. The discussion of these effects can be found in Carlson et al., Albaugh, and Wallis. ${ }^{4,5,14}$

Based on the earlier reasoning, we modified our theory to take into account of the reduction of electric stress by replacing $V$ in Eq. (20b) by $V_{\text {eff }}$. The magnitude of $V_{\text {eff }}$ is unknown which can only be determined by experiment. The collapse condition is obtained by replacing $V$ by $V_{\text {eff }}$ in Eq. (28), i.e.,

$$
E_{\text {eff }} d^{3} / \epsilon_{a} V_{\text {eff }}^{2} h_{g}=a_{c} / h_{g} .
$$

For our case, $V_{\text {eff }}$ is found to be $V / 2$. Equation (29) is plotted in Fig. 9 as a dashed line. It is clear that this modification provides a much better fit to the experimental data.

\section{DISCUSSION AND SUMMARY}

It is interesting to compare the present analysis with that of Anthony's which models the glass layer as an elastic beam. ${ }^{6}$ The condition for the collapse in this case is easily shown to be

$$
E_{\text {eff }} d_{\text {Beam }}^{3} /\left(h_{g} \epsilon_{a} V^{2}\right)=\left(a / h_{g}\right)^{4} / 4 .
$$

Equation (30) is plotted as a dotted line in Fig. 9. It can be seen that Eq. (25) and our modified Eq. (29) provide a much more accurate prediction of the contact criterion for microchannels. In addition, the scaling based on the beam theory is inconsistent with the experimental data.

The problem of the channel collapse is intimately connected with anodic bonding. In anodic bonding, the goal is to achieve good contact between two slightly nonconforming surfaces. The nonconformity is due to surface roughness which creates air gaps or cavities between asperities along the bonding interface. Eliminating these cavities is a necessary first step for good bonding. The process of the cavity collapse is similar to the collapse of microchannels illustrated in Fig. 8. The first step of this process involves contact of the cavity wall. Once in contact, it collapses quickly under the influence of attractive interfacial surface forces. A detailed discussion of the effect of surface forces on increasing the contact area of nonconforming surfaces can be found in Hui et al. ${ }^{15,16}$ and the literature within these papers. From this discussion, the anodic bonding process imposes a lower limit on the size of the microchannels. In other words, for the 
same aspect ratio, the height of the microchannel must be large compared with $d_{r}$, the mean surface roughness or cavity height.

A very rough estimation for $t_{\text {end }}$ in anodic bonding can be obtained by replacing $d$ and $a$ in the right-hand side of Eq. (21) by $d_{r}$ and $a_{m}$, respectively, where, $a_{m}$ is the mean radius of the cavities

$$
t_{\mathrm{end}}=\left(\eta d_{r}^{3} / a_{m} \epsilon_{a} V^{2}\right)\left[1-\left(\epsilon_{a} V^{2} a_{m} / E_{\mathrm{eff}} d_{r}^{3}\right)\right] .
$$

It should be noted that Eq. (31) does not take account of the time needed for complete contact, so that it underestimates $t_{\text {end }}$. However, once contact occurs, the two opposing surface rapidly bond, so we expect $t_{\text {end }}$ to be a good estimate of the time required for bonding.

Equation (31) also provides a criterion for the elastic collapse of the glass plate on the silicon during anodic bonding. Indeed, Eq. (31) implies that contact occurs when

$$
V>d_{r} \sqrt{E_{\mathrm{eff}} d_{r} / \epsilon_{a} a_{m}} .
$$

Equation (32) differs considerably from the criterion proposed by Anthony. ${ }^{6}$ Anthony's criterion, in the notation of the present article, is

$$
V>\sqrt{E_{\mathrm{eff}} h_{g}^{3} d_{r}^{3} / 80 a_{m}^{4} \epsilon_{a}} .
$$

Because the surface roughness is typically much smaller than all relevant length scales encountered in the problems, the critical voltage for the elastic contact is expected to be independent of the glass thickness. The elastic contact condition (32) is consistent with this reasoning, whereas in Anthony's criterion, the critical voltage for the elastic collapse is proportional to $h_{g}^{3 / 2}$. This is because Anthony assumes that the glass layer can be modeled as a beam. This assumption is incorrect for the following reason: the height and width of the surface relief (beam length) is typically much smaller than the thickness of the Pyrex glass (beam height), so that the usual beam theory is violated. Anthony's criterion is appropriate if the thickness of the Pyrex glass in much less than the width of the surface relief. Since this is typically not the case, we account for local deformation of the surfaces by modeling the glass layer as infinite. As an example, consider the example of Anthony, ${ }^{6}$ where $d_{r}=2 \times 10^{-6} \mathrm{~m}, a_{m}$ $=2 \times 10^{-5} \mathrm{~m}, E_{\text {eff }}=6 \times 10^{11} \mathrm{~Pa}$, and $\epsilon_{a}=8.85 \times 10^{-13} \mathrm{~F} / \mathrm{m}$. According to Eq. (32), the voltage required for the complete elastic contact of the Pyrex plate on the silicon is about $2 \times 10^{5} \mathrm{~V}$ whereas according to Anthony's criterion (33), this voltage is about $2 \times 10^{6} \mathrm{~V}$. Anthony's critical voltage is three times higher if the glass thickness is increased by a factor of 2. We also note that there is an error in Anthony's calculation. The critical voltage reported in his work is $150 \mathrm{~V}$ (instead of the $2 \times 10^{6} \mathrm{~V}$ found using his formula). We have not been able to precisely locate the source of this algebraic error (a likely scenario is that $a_{m}^{2}$ is used in the numerical calculation instead of $a_{m}^{4}$ ).

In summary, we have carried out a detailed electromechanical analysis to establish a criterion for the microchannel collapse. The collapse condition is independent of the thickness of the glass and the silicon wafer as well as the dielectric constants of the silicon and glass. The experiment results, which were obtained by anodic bonding microchannels with various aspect ratios, show good agreement with our analysis. We demonstrated that this collapse criterion can be used to aid in the design and fabrication of microchannels. We have also applied our results to obtain a rough estimation for the time needed to complete anodic bonding.

\section{ACKNOWLEDGMENTS}

W.P.S. and C.Y.H. would like to thank Professor Harold G. Craighead for bringing this problem to their attention and for various discussions during the initial stage of the project. The experiments were performed at Microfabrication Facility and MicroInstruments and System Laboratory (MISL), University of California at Davis. N.T. was supported by Berkeley Sensor and Actuator Center.

\section{APPENDIX}

Anodic bonding is typically carried out at $450{ }^{\circ} \mathrm{C}$, although a lower bonding temperature has been used. ${ }^{17}$ The later analysis considers the full temperature range. The surface potential of the silicon $\Phi_{s}$ is controlled by the doping concentration $N_{A}$, the absolute temperature $T$, and the concentration of intrinsic carriers $n_{i}$. At high temperatures $n_{i}$ $\gg N_{A}$ whereas $n_{i} \ll N_{A}$ at low temperature. $n_{i}$ in silicon is given by ${ }^{18}$

$$
n_{i}\left(\mathrm{~cm}^{-3}\right)=n_{0} T^{3 / 2} e^{-E_{\mathrm{gap}} / 2 k T}
$$

where $E_{\text {gap }}=E_{0}-A T^{2} /(B+T)$ and $k$ is the Boltzmann constant. The material constants in Eq. (A1) are given by $n_{0}$ $=7.3 \times 10^{15} \mathrm{~cm}^{-3}, E_{0}=1.17 \mathrm{eV}, A=4.73 \times 10^{-4} \mathrm{eV} / \mathrm{K}$, and $B=636 \mathrm{~K}$. A transition temperature $T_{\text {tran }}$, below which the intrinsic carrier plays a subordinate role, can be defined by setting $n_{i}=N_{A}$ in Eq. (A1), i.e.,

$$
n_{0} T_{\text {tran }}^{3 / 2} e^{-E_{\mathrm{gap}} / 2 k T_{\text {tran }}}=N_{A} .
$$

The dependence of $T_{\text {tran }}$ on $N_{A}$ is shown in Fig. 11, which indicates that bonding is typically carried out near the transition temperature under regular doping concentration $\left(10^{15}-10^{18} \mathrm{~cm}^{-3}\right)$ of silicon substrate. Therefore, the dopants and the intrinsic carriers both contribute to the surface potential of the silicon during bonding.

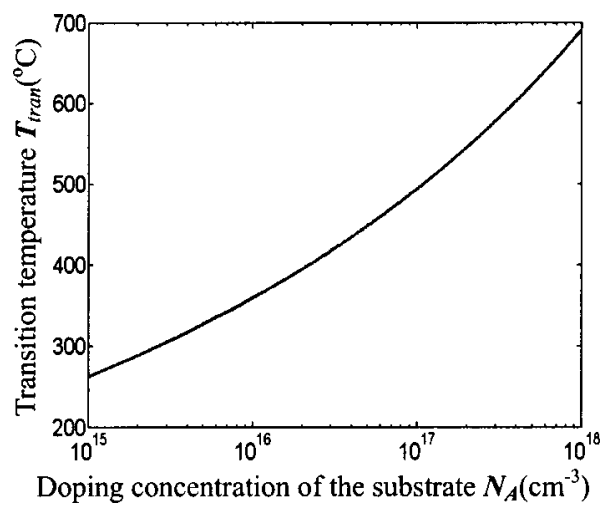

FIG. 11. Dependence of transition temperature $T_{\text {tran }}$ on the doping concentration $N_{A}$ of the $p$-type silicon wafer. 


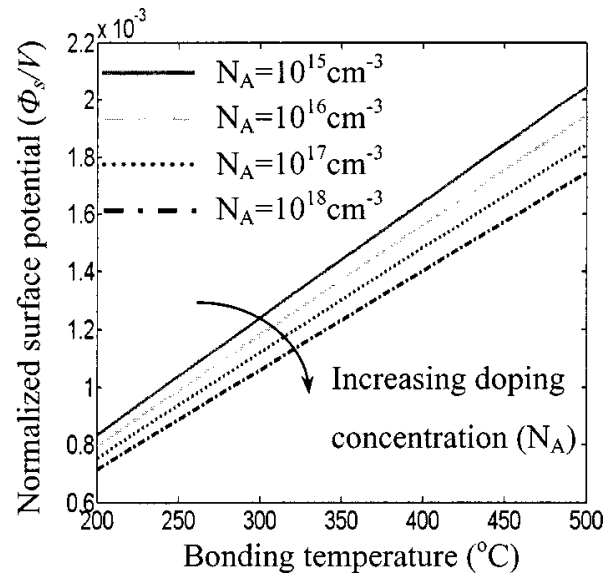

FIG. 12. Dependence of the normalized surface potential of the silicon $\Phi_{s} / V$ on doping concentration $N_{A}$ and bonding temperature.

Assume the microchannel is fabricated on a $p$-type silicon, the majority carrier concentration $p$ can be estimated using

$$
p \cong N_{A} e^{-q \Phi / k T}+n_{i},
$$

where $q$ is the absolute value of the electron charge. The first term in Eq. (A3) is due to band bending. The equilibrium minority carrier concentration $n$ is

$$
n=n_{i}^{2} / p=n_{i}^{2} /\left(N_{A} e^{-q \Phi / k T}+n_{i}\right) .
$$

Charge neutrality implies that the ionized doping concentration in the bulk silicon is

$$
N_{A}^{-}=p_{0}-n_{0}=N_{A}+n_{i}-n_{i}^{2} /\left(N_{A}+n_{i}\right),
$$

where the subscript " 0 " indicates that no band bending occurs in the bulk silicon. The potential $\Phi$ in silicon is determined using Poisson's equation, i.e.,

$$
\begin{aligned}
d^{2} \Phi / d y^{2}= & -\left(q / \epsilon_{s}\right)\left(p-n-N_{A}^{-}\right) \\
= & -\left(q n_{i} / \epsilon_{s}\right)\left\{r\left(e^{-q \Phi / k T}-1\right)+(1+r)^{-1}\right. \\
& \left.\times\left[1-M\left(N_{A} e^{-q \Phi / k T}+n_{i}\right)^{-1}\right]\right\},
\end{aligned}
$$

where $r \equiv N_{A} / n_{i}$ and $\boldsymbol{\epsilon}_{s}$ is the permittivity of silicon. Equation (A6) can be integrated to give

$$
\begin{aligned}
E_{s}^{2}= & \frac{2 k T n_{i}}{\epsilon_{s}}\left[r\left(e^{-q \Phi_{s} / k T}+\frac{q \Phi_{s}}{k T}-1\right)+\ln \left(\frac{r+e^{q \Phi_{s}} / k T}{r+1}\right)\right. \\
& \left.-\frac{q \Phi_{s}}{k T(r+1)}\right] .
\end{aligned}
$$

The surface charge density $q_{s}$ is, according to Gauss's law

$$
\begin{aligned}
q_{s}=\epsilon_{s} E_{s}= & \sqrt{2 k T \epsilon_{s} n_{i}}\left[r\left(e^{-q \Phi_{s} / k T}+\frac{q \Phi_{s}}{k T}-1\right)\right. \\
& \left.+\ln \left(\frac{r+e^{q \Phi_{s} / k T}}{r+1}\right)-\frac{q \Phi_{s}}{k T(r+1)}\right]^{1 / 2} .
\end{aligned}
$$

The relation between the maximum surface potential and the surface charge density $q_{s}$ of the silicon wafer is found by noting that $\Phi_{s}$ reaches its maximum when ion flow vanishes or when $d \delta / d \tau=0$. Using Eq. (10) with $d \delta / d \tau=0$, we found

$$
-2\left(1-\phi_{s}\right)+\alpha \delta_{\max }^{2}+2 \alpha \delta_{\max } / \beta=0,
$$

where $\delta_{\max }=-q_{s} / h_{g} \sigma_{p}$ is the maximum normalized thickness of the polarized layer. It is determined using Eq. (7b). The dependence of the maximum surface potential on $N_{A}$ and $T$ is found by solving Eq. (A9) together with Eq. (A8), with $n_{i}$ given by Eq. (A1). These results are shown in Fig. 12 for $\epsilon_{a}=8.85 \times 10^{-12} \mathrm{~F} / \mathrm{m}, \sigma_{p}=-2.72 \times 10^{8} \mathrm{C} / \mathrm{m}^{3}, d=100 \mathrm{~nm}$, $\epsilon_{g}=10 \epsilon_{a}, \epsilon_{s}=11.7 \epsilon_{a}$, and $V=-10^{3} \mathrm{~V}$. Figure 11 shows that the maximum value of $\phi_{s}=\Phi_{s} / V$ is on the order of $10^{-3}$. Therefore, neglecting $\phi_{s}$ in Eq. (10) is an excellent approximation for all temperatures and doping concentrations. In other words, the electric field across the air gap can be computed by treating the semiconductor as a perfect conductor with $\Phi_{s}=0$.

${ }^{1}$ J. Han and H. G. Craighead, Science 288, 1026 (2000).

${ }^{2}$ D. I. Pomerantz, US Patent No. 3,397,287 (1968), US Patent No. 3,417,459 (1968).

${ }^{3}$ D. E. Carlson, J. Am. Ceram. Soc. 57, 291 (1974).

${ }^{4}$ D. E. Carlson, K. W. Hang, and G. F. Stockdale, J. Am. Ceram. Soc. 55, 337 (1972).

${ }^{5}$ K. B. Albaugh, J. Electrochem. Soc. 138, 3089 (1991).

${ }^{6}$ T. R. Anthony, J. Appl. Phys. 54, 2419 (1983).

${ }^{7}$ J. A. Plaza, J. Esteve, and E. Lora-Tamayo, J. Electrochem. Soc. 144, 108 (1997).

${ }^{8}$ Y. C. Fung, Foundations of Solid Mechanics (Prentice Hall, Englewood Cliffs, NJ, 1965).

${ }^{9}$ P. M. Sutton, J. Am. Ceram. Soc. 47, 188 (1964).

${ }^{10}$ E. H. Snow and M. E. Dumesnil, J. Appl. Phys. 37, 2123 (1966).

${ }^{11} \mathrm{~J}$. R. Rice, in Mathematical Analysis in the Mechanics of Fracture, edited by Liebowitz (Academic Press, New York, 1968), Vol. 2, p. 192.

${ }^{12}$ L. H. Van Vlack, Physical Ceramics for Engineers (Addison Wesley, Reading, MA, 1964).

${ }^{13}$ Corning Inc., NY.

${ }^{14}$ G. Wallis, J. Amer. Ceram. Soc. 53, 277 (1970).

${ }^{15}$ C. Y. Hui, Y. Y. Lin, J. M. Baney, and E. J. Kramer, J. Polym. Sci., Part B: Polym. Phys. 39, 1195 (2001).

${ }^{16}$ C. Y. Hui, Y. Y. Lin, and J. M. Baney, J. Polym. Sci., Part B: Polym. Phys. 38, 1485 (2000).

${ }^{17}$ G. Wallis and D. I. Pomerantz, J. Appl. Phys. 40, 3946 (1969).

${ }^{18}$ F. J. Morin and J. P. Maita, Phys. Rev. 96, 28 (1954). 